\title{
Commento editoriale alle linee guida britanniche sull'ipertensione arteriosa 2006: "Hypertension: management of hypertension in adults in primary care. Partial update"
}

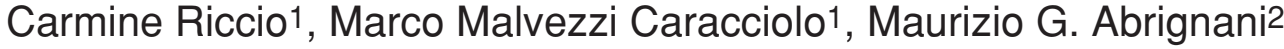

\author{
Monaldi Arch Chest Dis 2006; 66: 238-240. \\ 1 U.O. di Cardiologia Riabilitativa del Dipartimento di Scienze Cardiologiche, A.O. S. Sebastiano di Caserta. \\ 2 A.O. S. Antonio Abate, Erice Casa Santa (Trapani).
}

Dalla pubblicazione delle ultime linee guida sull'ipertensione arteriosa dell'European Society of Cardiology/European Society of Hypertension [1] e del VIIth Joint National Committee on Prevention, Detection, Evaluation, and Treatment of High Blood Pressure [2] sono ormai trascorsi tre anni, nel corso dei quali sono stati pubblicati diversi nuovi trial sul trattamento antiipertensivo, come ad esempio l'Anglo-Scandinavian Cardiac Outcomes Trial - Blood Pressure Lowering Arm (ASCOT-BPLA) [3], il Japan Multicenter Investigation for Cardiovascular Diseases-B (JMIC-B) [4], il Plaque Hypertension Lipid-Lowering Italian Study (PHYLLIS) [5] ed il Valsartan Antihypertensive Long-term Use Evaluation (VALUE) [6]. Alla luce dei dati emersi da questi e da altri studi, la comunità scientifica internazionale era pertanto in attesa di nuove linee guida sul trattamento antiipertensivo.

È giunta per prima al traguardo la British Hypertension Society (BHS), che, in collaborazione con il National Institute for Health and Clinical Excellence (NICE), il National Collaborating Centre for Chronic Conditions (organismo del NICE preposto alle linee guida), la Primary Care Cardiovascular Society ed il Royal College of Physicians, ha edito sul web il 28 giugno un aggiornamento, limitatamente al solo trattamento farmacologico, alle linee guida britanniche BHS [7] e NICE (clinical guideline 18) [8] sull'ipertensione arteriosa, pubblicate nel 2004. La decisione fa seguito ad una review preliminare condotta da un Expert Advisory Committee comprendente rappresentanti del NICE, della BHS e della Blood Pressure Association, un'associazione di pazienti inglesi. Alla base di questo approccio collaborativo vi è l'esigenza di evitare una duplicazione non necessaria degli sforzi degli esperti e di ridurre i potenziali rischi di confusione tra medici e pazienti.

Si tratta della prima volta che una società scientifica come la BHS, che da venti anni produce linee guida sull'ipertensione arteriosa, collabora con il NICE, un organismo governativo qualificato ed indipendente che ha il fine di fornire indicazioni nazionali sulla promozione della salute e la cura delle patologie, raggiungendo un ampio consenso nell'elaborazione delle stesse linee guida. Da segnalare anche il fatto che le linee guida NICE non vengono di solito aggiornate prima di quattro anni dalla pubblicazione.
Il lavoro, basato su una revisione sistematica su EMBASE e MEDLINE, attuata usando il software RevMan 4.2 (@The Nordic Cochrane Centre), dei dati pubblicati di recente in letteratura in 20 trial di confronto tra diversi trattamenti antiipertensivi, stabilisce il gold standard per un ottimale gestione farmacologica dell'ipertensione arteriosa, con lo scopo finale di ridurre la mortalità e morbilità associate a malattie cardiovascolari come l'ictus, l'insufficienza renale cronica e la cardiopatia ischemica, per le quali l'ipertensione è un fattore di rischio fortemente significativo.

L'importanza dell'argomento consegue anche alle dimensioni epidemiologiche della condizione, che coinvolge circa il $40 \%$ degli adulti in Gran Bretagna come negli altri paesi industrializzati (di cui un terzo ne è inconsapevole) ed è spesso trattata in modo inadeguato, nonostante che circa il $15 \%$ delle spese annuali per le cure primarie in Gran Bretagna sia relativo al trattamento antiipertensivo.

Il Guideline Development Group, presieduto dal dott. B. Higgins, nel formulare le raccomandazioni, ha preso in considerazione, sulla scorta dei principi dell'Evidence Based Medicine, non soltanto gli studi più recenti, ma anche i dati sugli effetti indesiderati e sui meccanismi d'azione delle differenti classi di antiipertensivi nelle diverse età e nei differenti ceppi etnici.

E stato anche analizzato un dettagliato studio di economia sanitaria sui rapporti costo-efficacia delle principali classi di agenti antiipertensivi, che ha raggiunto una conclusione simile a quella della metanalisi, e cioè che calcioantagonisti e diuretici appaiono le classi col miglior rapporto costo/benefici e che i betabloccanti sono quelli con gli effetti meno favorevoli. Da questa analisi si deduce che i migliori rapporti costo/efficacia dei farmaci antiipertensivi conseguono ai loro effetti relativi alla prevenzione del diabete mellito e dello scompenso cardiaco. In particolare, i calcioantagonisti sarebbero da preferire nei soggetti a basso rischio di scompenso cardiaco, ed i diuretici in quelli a basso rischio di diabete. Infine, nei soggetti a rischio sia di diabete che di scompenso cardiaco, i farmaci agenti sul sistema renina-angiotensina rappresenterebbero, dal punto di vista farmacoeconomico, l'opzione più favorevole. Da segnalare che l'applicabilità ai soggetti d'età inferiore a 55 anni del suddetto modello è incerta, in quanto basato su trial condotti su soggetti molto più anziani. 
Le principali raccomandazioni incluse nelle linee guida, che nel loro insieme rappresentano un autorevole e condiviso algoritmo nazionale di trattamento antiipertensivo, sono le seguenti:

- Negli ipertesi d'età superiore ai 55 anni e nei pazienti di colore (neri africani e dei Caraibi) di ogni età la terapia iniziale di scelta dovrebbe essere un calcioantagonista o un diuretico tiazidico (livello di evidenza A).

- Negli ipertesi più giovani la terapia iniziale di prima scelta dovrebbe essere un ACE-inibitore (o un sartano nel caso questo non sia tollerato) (livello di evidenza C).

- Nel caso sia necessario un secondo farmaco, ci si dovrebbe orientare verso l'uso di un ACE-inibitore (o un sartano nel caso questo non sia tollerato) nei pazienti già trattati con un calcioantagonista o un diuretico tiazidico, e verso un calcioantagonista o un diuretico tiazidico nei pazienti in trattamento con un farmaco inibente il sistema renina-angiotensina (livello di evidenza B).

- Se è necessario un trattamento con tre farmaci, si consiglia l'associazione tra ACE-inibitore (o un sartano nel caso questo non sia tollerato), un calcioantagonista ed un diuretico tiazidico (livello di evidenza B).

- Se la pressione è ancora sopra il target di 140/90 $\mathrm{mmHg}$, si suggerisce di aggiungere un quarto farmaco o di consultare uno specialista (livello di evidenza $\mathrm{C}$ ).

- Se si decide di utilizzare un quarto farmaco, si può incrementare la dose di diuretico tiazidico o prescrivere un altro diuretico (raccomandando un attento monitoraggio), un betabloccante od un alfa bloccante selettivo (livello di evidenza C).

- Se ciononostante la pressione arteriosa rimane non controllata dovrebbe essere consultato uno specialista (livello di evidenza C).

- I betabloccanti non sono più indicati come terapia di prima scelta per l'ipertensione arteriosa. Tuttavia il loro impiego può essere preso in considerazione nei giovani (livello di evidenza B), particolarmente se in presenza di controindicazioni ai farmaci interagenti sull'asse renina-angiotensina, nelle donne in età fertile o nei pazienti che manifestano un'iperattività simpatica. In questi casi, se s'intraprende una terapia con betabloccanti e si rende necessario un altro farmaco, si consiglia di aggiungere un calcioantagonista e non un diuretico allo scopo di ridurre il rischio d'insorgenza di diabete mellito (livello di evidenza C).

- Nei pazienti in cui la pressione arteriosa non è controllata con un regime terapeutico che include un betabloccante, la terapia andrebbe rivista secondo le suddette raccomandazioni (livello di evidenza C).

- Nei pazienti in cui il trattamento antiipertensivo comprendente un betabloccante sia sufficiente a raggiungere il target pressorio $(<140 / 90 \mathrm{~mm} \mathrm{Hg})$ non vi è necessità di sostituire il betabloccante con un altro farmaco (livello di evidenza C).

- Quando un betabloccante deve essere sospeso, il dosaggio deve essere ridotto gradatamente.
Inoltre i betabloccanti non devono essere sospesi in pazienti con indicazioni elettive, come ad esempio i pazienti coronaropatici con angina o con pregresso infarto miocardico (livello di evidenza C).

Questo nuovo algoritmo sul trattamento antiipertensivo contrasta notevolmente con le precedenti linee guida NICE [4], in cui si consigliava di iniziare la terapia con un diuretico aggiungendo un betabloccante e successivamente, come terza scelta, un calcioantagonista. Gli ACE inibitori venivano considerati in alternativa ai betabloccanti nei pazienti ad alto rischio di insorgenza di diabete o come quarta scelta nei pazienti non responders a betabloccanti, diuretici e calcioantagonisti.

La decisione di non raccomandare l'uso dei betabloccanti come prima scelta è probabilmente l'aspetto più innovativo (e discusso) di queste linee guida ed è basata sui risultati di recenti trial comparativi [3-6] e di metanalisi come quella di Lindholm [9], che suggeriscono che il loro effetto sia inferiore a quello degli altri farmaci, particolarmente nell'anziano e nella prevenzione dell'ictus, nonché sull'evidenza del rischio di insorgenza di diabete mellito (ma anche di dislipidemia) in seguito al trattamento coi betabloccanti più comuni alle dosi usuali. Inoltre gli importanti effetti collaterali dei betabloccanti, come astenia, impotenza, sonnolenza, parestesie agli arti e sbalzi d'umore possono compromettere significativamente la qualità di vita del paziente riducendone la compliance ai trattamenti.

Un'obiezione che potrebbe essere avanzata nei confronti di quest'impostazione è che i trial in questione, come l'ASCOT [3] ed il LIFE [10], hanno preso in considerazione un solo betabloccante, seppure il più usato, l'atenololo, per cui non vi sono dati disponibili sugli altri farmaci della stessa classe. Inoltre tutti gli studi presi in considerazione erano stati condotti su pazienti anziani, in cui notoriamente i betabloccanti non sono così efficaci quanto i tiazidici od i calcioantagonisti; infine, molti di questi studi hanno usato l'atenololo in monosomministrazione, probabilmente un dosaggio non ottimale per un betabloccante. Il rischio maggiore che si potrebbe verificare è che, stante la larga diffusione di queste linee guida anche tra i non addetti ai lavori, si possa instaurare una "psicosi da betabloccanti", che porti i pazienti ad abbandonare, magari bruscamente e senza parere medico, farmaci in realtà essenziali per le loro condizioni cliniche.

Se i betabloccanti vengono scalzati dal loro trono in una delle loro roccaforti migliori, questo invece non accade ai diuretici, anche se forse sarebbe stato opportuno sottolineare l'opportunità di evitare il loro uso nei pazienti a rischio di diabete mellito di nuova insorgenza, come nei pazienti con sindrome metabolica [11].

La collocazione dei sartani come alternativa nel caso gli ACE-inibitori non siano tollerati è probabilmente il risultato di alcuni dati emersi da studi come il LIFE [10], in cui il rischio di ictus è stato ridotto dal trattamento con il Losartan in confronto ad atenololo più diuretico ma in assenza di effetti sugli endpoint infarto miocardico e morte cardiovascolare, ed il VALUE [6], in cui si è registrato un incremento del 19\% del rischio di infarto miocardico. 
Probabilmente ci si poteva aspettare da queste linee guida una raccomandazione più incisiva sulla possibilità di iniziare, quantomeno nei pazienti ad alto rischio, il trattamento con una combinazione di due farmaci, dato che il $90 \%$ dei pazienti del'ASCOT erano in questa condizione, ma è rilevante il richiamo al raggiungimento dei valori tensivi di $140 / 90 \mathrm{mmHg}$, come già suggerito dalle precedenti linee guida internazionali $[1,2]$ e dai dati del Blood Pressure Lowering Treatment Trialists' Collaboration [12], che hanno dimostrato che solo in questo caso ci si può aspettare una riduzione degli eventi cardiovascolari.

Da questo punto di vista molte delle riserve che si sono via via avanzate sulle varie classi di agenti antiipertensivi vengono superate dall'esigenza di raggiungere questo target, cosa possibile il più delle volte solo con l'associazione di più farmaci. L'associazione farmacologica più efficace e con i minori effetti indesiderati, secondo queste linee guida, appare quella tra calcioantagonista, diuretico tiazidico ed ACE inibitore/sartano.

È importante anche che venga sottolineata la validità di tutti gli altri aspetti delle linee guida originali che coprono tutti gli aspetti della gestione della patologia ipertensiva come gli interventi sullo stile di vita, evidenziando come cruciale un appropriato approccio olistico.

È auspicabile che anche queste linee guida, notevoli per la loro semplicità, chiarezza, e facilità di implementazione nella pratica clinica, siano utili nello stimolare i medici a rivedere il trattamento dei loro pazienti ipertesi allo scopo di raggiungere i valori target di pressione arteriosa nel maggior numero possibile di essi. I messaggi chiari e rassicuranti rivolti ai pazienti (le linee guida sono corredate da ampie e chiare Informazioni per i Pazienti) li potranno aiutare ad avvantaggiarsi dell'ampia disponibilità di sistemi di monitoraggio domiciliare e migliorare la comunicazione con i loro curanti per divenire partner informati ed attivi in questo processo.

Diverse versioni delle linee guida sono disponibili nel sito web della NICE: una più completa, Clinical guideline 34: Hypertension: management of hypertension in adults in primary care: partial update [13]; le raccomandazioni "Hypertension: management of hypertension in adults in primary care" [14]; una Quick reference guide [15]; ed infine le informazioni per i pazienti "Understanding NICE guidance" [16].

\section{Bibliografia}

1. Guidelines Committee. 2003 European Society of Hypertension - European Society of Cardiology guidelines for the management of arterial hypertension. J Hypertension 2003, 21: 1011-1053.

2. Chobanian AV, Bakris GL, Black HR, et al. Seventh Report of the Joint National Committee on Prevention, Detection, Evaluation, and Treatment of High Blood Pressure. Hypertension 2003; 42: 1206.

3. Dahlöf B, Sever P, Poulter N, et al. Prevention of cardiovascular events with an antihypertensive regimen of amlodipine adding perindopril as required versus atenolol adding bendroflumethiazide as required, in the AngloScandinavian Cardiac Outcomes Trial-Blood Pressure Lowering Arm (ASCOT-BPLA): a multicentre randomised controlled trial. Lancet 2005; 366: 895-906.

4. Yui Y, Sumiyoshi T, Kodama K, et al. Comparison of nifedipine retard with angiotensin converting enzyme inhibitors in Japanese hypertensive patients with coronary artery disease: the Japan Multicenter Investigation for Cardiovascular Diseases-B (JMIC-B) randomized trial. Hypertension Res Clin Exptl 2004; 27: 181-91.

5. Zanchetti A, Crepaldi G, Bond MG, et al. Different effects of antihypertensive regimens based on fosinopril or hydrochlorothiazide with or without lipid lowering by pravastatin on progression of asymptomatic carotid atherosclerosis: principal results of PHYLLIS - a randomized double-blind trial. Stroke 2004; 35: 2807-12.

6. Julius S, Kjeldsen SE, Weber M, et al. Outcomes in hypertensive patients at high cardiovascular risk treated with regimens based on valsartan or amlodipine: the VALUE randomised trial. Lancet 2004; 363: 2022-31.

7. Williams B, Poulter NR, Brown MJ, et al. Guidelines for management of hypertension: report of the fourth working party of the British Hypertension Society, 2004-BHS IV. Journal of Human Hypertension 2004; 18: 139-85.

8. www.nice.org.uk/page.aspx $\mathrm{o}=\mathrm{cg} 018$ background.

9. Lindholm L, Carlberg B, Samuelsson O. Should betablockers remain first choice in the treatment of primary hypertension? A meta-analysis. Lancet 2005, 366: 1545-1553.

10. Kjeldsen SE, Lyle PA, Kizer JR, et al. The effects of losartan compared to atenolol on stroke in patients with isolated systolic hypertension and left ventricular hypertrophy. The LIFE study. J Clin Hypertension 2005; 7: 152-8.

11. Mancia G, Grassi G, Zanchetti A. New-onset diabetes and antihypertensive drugs. J Hypertens 2006; 24: 3-10.

12. Blood Pressure Lowering Treatment Trialists' Collaboration. Effects of different blood pressure-lowering regimens on major cardiovascular events in individuals with and without diabetes mellitus results of prospectively designed overviews of randomized trials. Arch Intern Med 2005; 165: 1410-1419.

13. www.nice.org.uk/CG034guidance

14. www.nice.org.uk/CG034NICEguideline

15. www.nice.org.uk/CG034quickrefguide

16. www.nice.org.uk/CG034publicinfo 